Int. J. Dev. Biol. 48: 771-782 (2004)

doi: $10.1387 / \mathrm{ijdb} .041894 \mathrm{cs}$

\title{
A re-examination of lens induction in chicken embryos: in vitro studies of early tissue interactions
}

\author{
CHARLES H. SULLIVAN ${ }^{1}$, LESLIE BRAUNSTEIN ${ }^{2}$, ROYCE M. HAZARD-LEONARDS ${ }^{2}$, ANNA L. HOLEN ${ }^{1}$, \\ FOUAD SAMAHA ${ }^{2}$, LAURIE STEPHENS ${ }^{2}$ and ROBERT M. GRAINGER ${ }^{* 2}$ \\ ${ }^{1}$ Department of Biology, Grinnell College, Grinnell, IA, USA and ${ }^{2}$ Department of Biology, University of Virginia, Charlottesville, VA, USA
}

\begin{abstract}
Early studies on lens induction suggested that the optic vesicle, the precursor of the retina, was the primary inducer of the lens; however, more recent experiments with amphibians establish an important role for earlier inductive interactions between anterior neural plate and adjacent presumptive lens ectoderm in lens formation. We report here experiments assessing key inductive interactions in chicken embryos to see if features of amphibian systems are conserved in birds. We first examined the issue of specification of head ectoderm for a lens fate. A large region of head ectoderm, in addition to the presumptive lens ectoderm, is specified for a lens fate before the time of neural tube closure, well before the optic vesicle first contacts the presumptive lens ectoderm. This positive lens response was observed in cultures grown in a wide range of culture media. We also tested whether the optic vesicle can induce lenses in recombinant cultures with ectoderm and find that, at least with the ectodermal tissues we examined, it generally cannot induce a lens response. Finally, we addressed how lens potential is suppressed in non-lens head ectoderm and show an inhibitory role for head mesenchyme. This mesenchyme is infiltrated by neural crest cells in most regions of the head. Taken together, these results suggest that, as in amphibians, the optic vesicle cannot be solely responsible for lens induction in chicken embryos; other tissue interactions must send early signals required for lens specification, while inhibitory interactions from mesenchyme suppress lens-forming ability outside of the lens area.
\end{abstract}

KEY WORDS: lens induction, chicken development, delta-crystallin, tissue interactions

Introduction

For nearly a century, induction of a lens has served as a model of the tissue interactions that occur during vertebrate development (Spemann, 1938; reviewed by Saha et al., 1989). While early experiments focused on the role of the optic vesicle (presumptive retina) in inducing a lens in overlying ectoderm, more recent experiments with amphibian embryos have questioned the role of the optic vesicle as the sole early inducing tissue (Henry and Grainger, 1987; Grainger et al., 1988; Henry and Grainger, 1990). In experiments testing the efficacy of the optic vesicle as an inducer, where donor and host tissues could be distinguished, the lenses that formed were not induced from host tissue, but originated from donor tissue (Grainger et al., 1988). Also, "free" lenses can form in the absence of other eye structures when the presumptive optic vesicle is surgically removed (reviewed by Saha et al., 1989). Based on these experiments, the classic, one-step model of lens induction by the optic vesicle has been revised. The major components of the newer model of lens induction are that the optic vesicle is not a sufficient lens inducer, but that an earlier lateral interaction between anterior neural plate and competent head ectoderm established a lens-forming bias in the latter tissue (reviewed by Grainger, 1992; 1996; Hirsch and Grainger, 2000). An inhibitory interaction between head mesenchyme and the head ectoderm is proposed to restrict the lens bias to the presumptive lens ectoderm. Experiments by von Woellwarth (1961) suggested that migrating neural crest cells could be responsible for this inhibition. He studied free lens formation in Triturus alpestris and observed a much higher proportion of embryos with lenses if neural crest precursors were removed along with the primordial of the optic vesicles. However, when neural crest cells were present, free lens formation was greatly reduced. The optic vesicle may still play a role in the last stages of lens determination $(H$. Jin and $R$.

Abbreviations used in this paper: DIC, differential interference contrast.

\footnotetext{
*Address correspondence to: Dr. Robert M. Grainger, Department of Biology, Gilmer Hall, University of Virginia, Charlottesville, VA 22904. Fax: +1-434-982-5626. e-mail: rmg9p@virginia.edu
} 


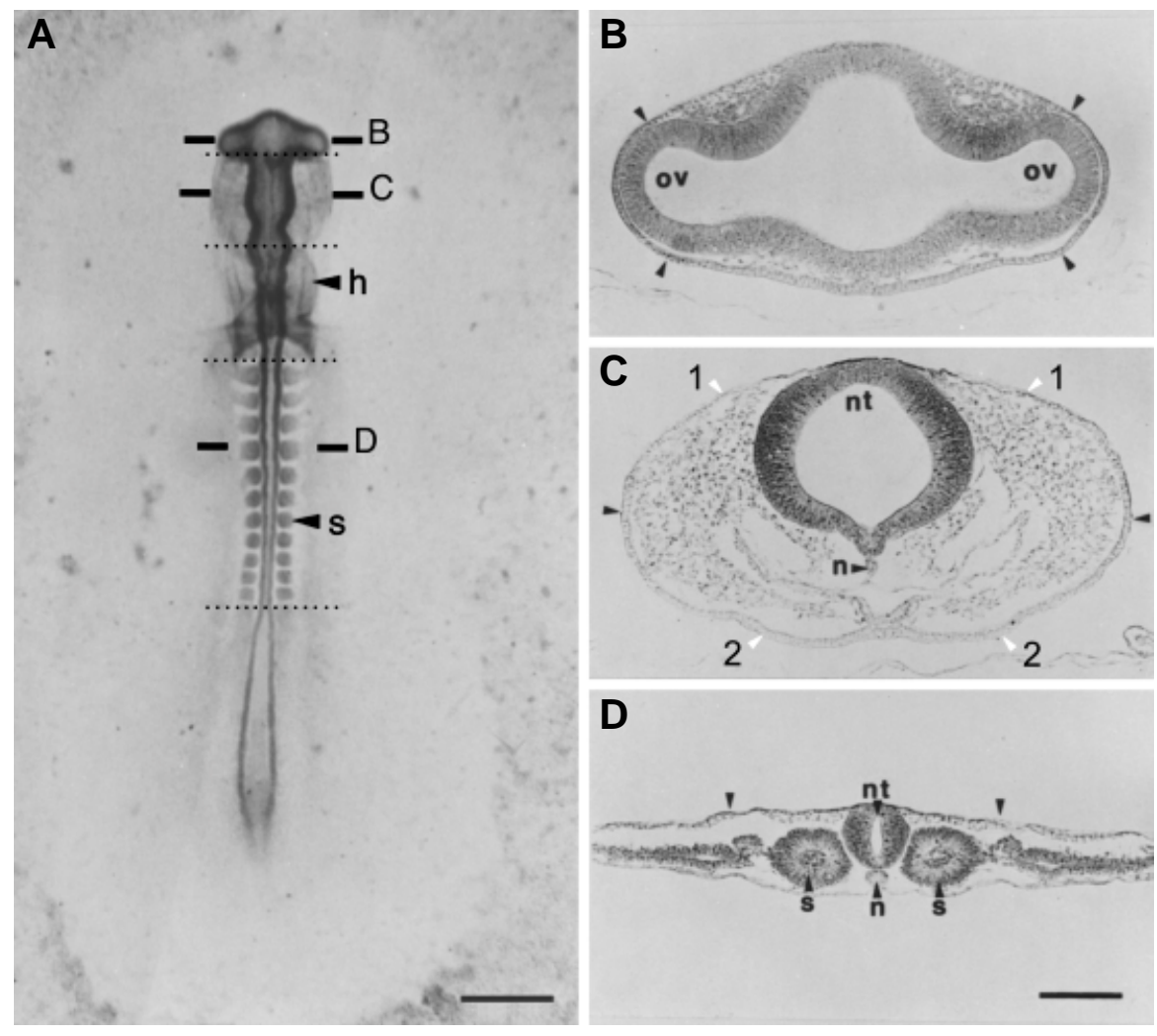

Fig. 1. Light micrographs of a stage $\mathbf{1 0}$ chicken embryo. Dotted lines (A) indicate where cuts were made to isolate different regions of the embryo to study lens-forming potential in the surface ectoderm. Solid lines indicate the positions of transverse sections through the (B) optic vesicles, (C) midbrain, and (D) trunk region shown to the right (dorsal surface is up). The heart ( $h$ ) and somites (s) are well formed by this stage. Black arrowheads show the boundaries of presumptive lens ectoderm collected from over the optic vesicles (ov) in (B); ventral ectoderm collected at the level of the midbrain and hindbrain in (C); and trunk ectoderm from over the neural tube (nt), notochord (n), and somites (s) in (D). Numbered white arrowheads indicate the boundaries of lateral head ectoderm and ventral medial ectoderm used for experiments in Fig. 7. Lateral head ectoderm extends from while arrowheads 1-2 on each side; ventral-medial ectoderm extends between white arrowheads 2-2. Scale bar, $0.5 \mathrm{~mm}$ in A; scale bar, $100 \mu \mathrm{m}$ in D for B, C, and D.

Grainger, unpublished) and also has an important role in lens differentiation (Coulombre and Coulombre, 1963).

Lens formation in other vertebrate species has not been studied in the same detail as in amphibians and has not been thoroughly reinvestigated since unambiguous molecular markers for the lens phenotype have become available. Several older experiments argued that lens induction by the optic vesicle begins at the 10somite stage in chicken embryos (stage 10 according to Hamburger and Hamilton, 1951; see Fig. 1) when the optic vesicle and presumptive lens ectoderm strongly adhere (McKeehan, 1951). When an optic vesicle or vesicle anlage was transplanted to a site on the head or body of a host embryo, morphological changes consistent with lens differentiation occurred in the area of the transplant (Alexander, 1937; McKeehan, 1951). Optic vesicles from a variety of stages were associated with ectopic lenses in host embryos. Second, when optic vesicles and trunk ectoderm were combined and grown in vitro for several days, lens-like structures were seen that stained with an anti-lens antibody, assayed by immunofluorescence, in over $60 \%$ of the cultures (KarkinenJääskeläinen, 1978). Together, these studies are consistent with the classic view that the optic vesicle is potent lens inducer in chicken embryos.

However, other studies suggest a primary role for earlier tissue interactions in the case of chick lens formation. Barabanov and Fedtsova (1982) showed that head ectoderm far away from influence of the optic vesicle will form a lens when isolated as early as the head process stage (stage 5) and grown in culture. They concluded that lens induction was a multi-step process that prepared the ectoderm for possible later inductive signals from the optic vesicle. Jorquera et al., (1989) present data suggesting that tissues other than the optic vesicle, namely cardiac mesoderm, are important in lens formation. Their studies agree with earlier work in amphibian embryos that demonstrated a role in lens induction for tissues that transiently contacts the presumptive lens ectoderm during gastrulation (Jacobson, 1966).

More recent molecular studies of lens formation are consistent with a model of lens induction that begins early in development in the chick. For example, the PaxG gene is essential for eye and lens formation and plays a role in early lens development (Ashery-Padan and Gruss, 2001; Lang, this issue). Pax6, which is an early response to lens induction (Zygar et al., 1998), is first detected in chicken embryos at stage 5 in head ectoderm (Li et al., 1994). In rats (Fujiwara et al., 1994) and mice (Enwright and Grainger, unpublished), this gene is necessary for the ectoderm to be fully responsive inductive signals. If Pax 6 has the same role in chicken embryos, then this is further evidence that lens induction begins much earlier than stage 10. Because there is apparently contradictory evidence regarding the mechanism of lens induction and the contribution of the optic vesicle in chicken embryos, and in order to test the universality of the amphibian model, we have re-examined this process.

Two other issues emerge that are relevant to the to the work presented here on chick lens induction. First, in order to study tissue interactions, it is necessary to have a specific assay for differentiation (Saha etal., 1989). Indeed, one possible explanation for the inconsistencies in previous studies of chick lens induction is the variety of assays for lens formation used by different investigators. Many of the older studies used cell elongation characteristic of lens fiber cells alone as an assay for a positive lens response (Alexander, 1937; McKeehan, 1951), yet tissue thickening is a common response to the surgical procedures used (see Saha et al., 1989). Later studies employed immunofluorescence using antibodies to the lens (Karkinen-Jääskeläinen, 1978) or to the major lens protein, delta-crystallin (Barabanov and Fedtsova, 1982). A potential complication with using delta-crystallin (and many other crystallins) as a marker of lens differentiation is that the messenger RNA for this protein has been detected in a variety of non-lens tissues in chicken embryos (Agata et al., 1983; Jeanny et 
al., 1985; Sullivan etal., 1991b). We find, however, that a combination of morphological changes associated with lens differentiation and the synthesis the delta-crystallin protein, confirmed by immunoblot analysis (Sullivan et al., 1998), provide a definitive assay for lens formation. Therefore, we have used immunoblot analysis complemented by histological assays to monitor lens cell differentiation in our studies designed to re-examine the role of the optic vesicle.

A second complication concerns the requirement that long-term cultures of chicken cells and tissues be grown in complex media that may contain growth factors and other components that could themselves affect inductive responses (Bronner-Fraser, 1996). Typically a balanced salt solution is supplemented with both serum and embryo extract to better mimic the conditions in vivo. A further complication is that different recipes for preparing culture media have been used in different investigations. In the present study we took two approaches when preparing media. In some cases we sought to repeat culture experiments as closely as possible, especially when re-examining the role of the optic vesicle as the lens inducing tissue (Karkinen-Jääskeläinen, 1978). In other cases we studied the lens response in a range of media conditions to get a sense of the potential effects of different components on induction.

In the current study we found that a large region of head ectoderm is specified for lens formation by stage 8 , even though close contact between the optic vesicle and presumptive lens ectoderm does not occur until stage 10 . In addition, specification of both presumptive lens ectoderm and head ectoderm is retained in a variety of culture media. These results led to a reinvestigation of the ability of stage 10 optic vesicles to induce lenses in trunk ectoderm. We found that optic vesicles induced lens formation in trunk ectoderm in fewer than $4 \%$ of the cultures at a variety of stages tested. We also observed that the optic vesicle cannot induce a lens response in stage 4 epiblast tissue. Finally, we found a role for head mesenchyme cells in suppressing the lens-forming bias in non-lens head ectoderm and observed that neural crest cells (positive for the marker HNK-1) are migrating into this mesenchyme during the period when the lens potential is being suppressed.

\section{Results}

\section{Spatial and temporal specification of lens fate}

As a starting point for evaluating the role of the optic vesicle, and possibly other tissues, in lens in induction in the chick we assessed the degree to which ectoderm in different regions of the embryo is specified for lens formation. Previously, Barabanov and Fedtsova (1982) reported that lens-forming ability existed in head ectoderm some distance from the optic vesicle and before the time of optic vesicle contact with lens ectoderm. Several ectodermal tissues were isolated from stage 10 embryos (Fig. 1) and cultured under the rich culture conditions used earlier by Karkinen-Jääskeläinen (1978) studying the role of the optic vesicle as a lens inducer. After five days of culture in media containing $20 \%$ embryo extract and $15 \%$ serum (medium A), tissues were examined for delta-crystallin synthesis on immunoblots (Fig. 2). Both presumptive lens ectoderm from over the optic vesicles and head ectoderm from the ventral surface of the midbrain and hindbrain were already specified for lens formation by stage 10 . Over $92 \%$ of cultures of presumptive lens ectoderm and $97 \%$ of cultures of ventral head ectoderm were

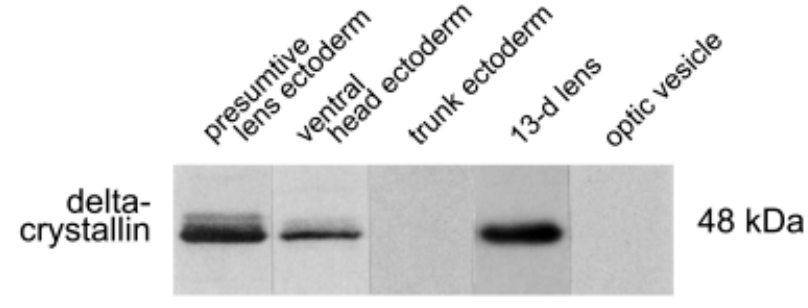

Fig. 2. Immunoblot analysis of delta-crystallin expression in different tissues. Explants of presumptive lens ectoderm, ventral head ectoderm, and trunk ectoderm were isolated and grown in culture for five days, then processed for electrophoresis. The antibody recognizes a $48 \mathrm{kDa}$ protein in a positive control from 13-day embryonic chick lenses and in presumptive lens ectoderm and ventral head ectoderm. No staining was observed in trunk ectoderm or optic vesicle (negative control).

positive (Fig. 3), while ectoderm from over the somites was always negative under identical culture conditions (Figs. 2 and 3). Thus, when using the immunoblot assay we confirmed the results of Barabanov and Fedtsova (1982) who used immunofluorescence and cultured their tissues in $10 \%$ embryo extract and $10 \%$ FCS. We also confirmed their observation that presumptive lens ectoderm is specified at stage $8 ; 100 \%$ of these cultures were positive $(n=8)$ when grown in medium A. Barabanov and Fedtsova (1982) also reported $75 \%$ of their cultures of stage 6 head ectoderm and $54 \%$ of stage 5 head ectoderm were positive for lens cells. However, in our experiments, at stage 4 none of the cultures was positive $(n=$ 18). In addition to our results using immunoblots, we examined the histology of each type of culture. Most of the cells in stage 10 presumptive lens ectoderm cultures were elongated and stained with the delta-crystallin antibody (Fig. 4 A,D). A substantially

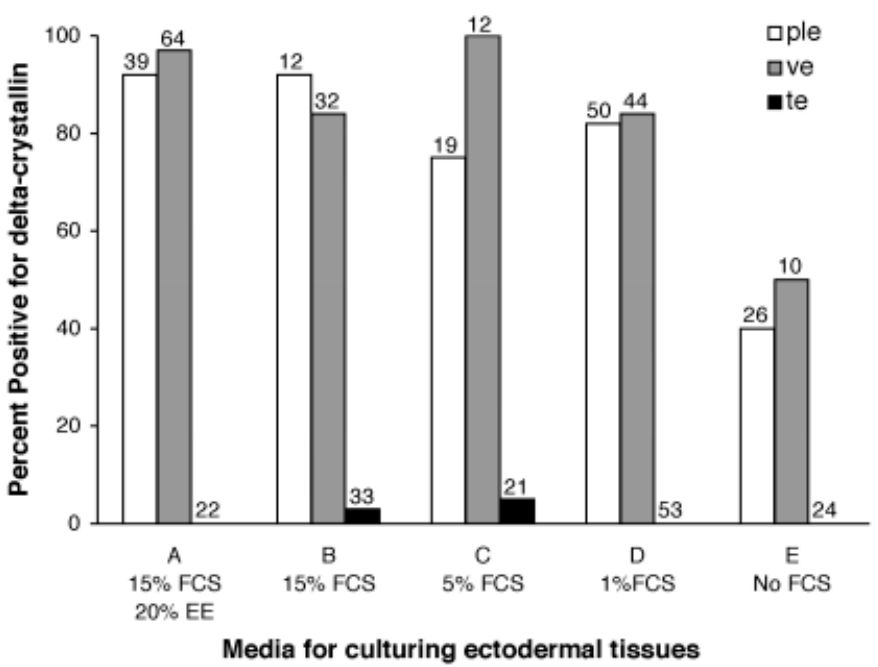

Fig. 3. Specification of different regions of ectoderm from stage 10 chicken embryos grown in different media. Ectoderm was removed by trypsinization of regions of the embryo shown in Fig. 1. Bars show the proportion of cultures that were positive on the basis of delta-crystallin synthesis as assayed on immunoblots. The number above each bar indicates the number of cases scored. 

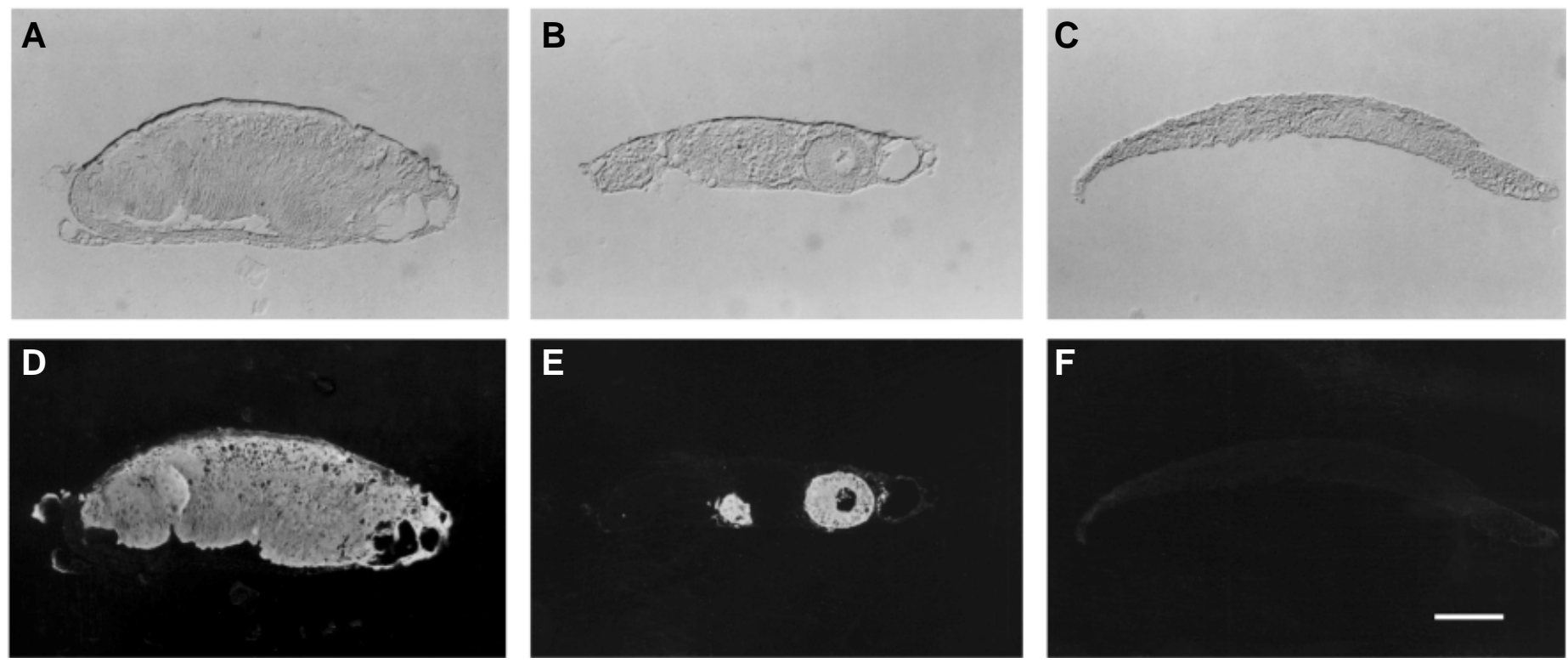

Fig. 4. Lens specification in vitro. Different regions of ectoderm from stage 10 chicken embryos were grown in Medium A for five days, then fixed. The top row (A-C) shows sections viewed with differential interference contrast (DIC); the bottom row (D-F) shows immunofluorescence with a delta-crystallin antibody and a fluorescein-labeled secondary antibody. Explants of presumptive lens ectoderm $(A, D)$, ventral head ectoderm (B, E) and trunk ectoderm (C, $F)$ were tested for a lens response by antibody staining. Scale bar, $100 \mu \mathrm{m}$.

smaller region of cells in cultures of ventral head ectoderm contained delta-crystallin-positive, elongated cells (Fig. 4 B,E). However, cultures of trunk ectoderm did not contain any elongated cells and were negative for antibody staining (Fig. 4 C,F).

The experiments in the previous section revealed that a subset of cells in the head ectoderm surrounding the midbrain and hindbrain were capable of expressing delta-crystallin after several days of growth in vitro. Apparently cells in this head ectoderm have received early signals for lens formation. To determine the extent of specification in the head region, five additional regions of anterior ectoderm from ventral and dorsal sides of stage 10 embryos were isolated and cultured (Fig. 5, Table I). A positive lens response was seen in $83 \%$ of the cultures of anterior ectoderm from the ventral surface of the forebrain between the two optic vesicles (region 1 on Fig. 5A). Ectoderm from the dorsal side of the forebrain between the optic vesicles (region 3 on Fig. 5B) showed a weaker response with only $18 \%$ of the cultures positive. Ectoderm from the dorsal surface of the head posterior to the optic vesicles and anterior to heart (region 4) was also positive in only $18 \%$ of the cases. Cultures of ectoderm taken from the level of the heart from either the ventral surface (region 2) or the dorsal surface (region 5 ) were rarely positive. These results revealed the strongest lens response in cultures of anterior ectoderm and a higher specification on the ventral surface of the forebrain than on dorsal surface (Table I). Although we have not verified that signals have been received by the specified ectoderm, we do note that the regions of head ectoderm tested express a differential lens response as has been noted when testing the lens forming bias of Xenopus head ectoderm (Grainger et al., 1997).

Based on earlier work on Pax6 transcription in chicken tissues during this period (Li et al., 1994), we decided to examine whether the spatial patterns of specification correlated with expression of the Pax6 protein. When whole mounts of chicken embryos were

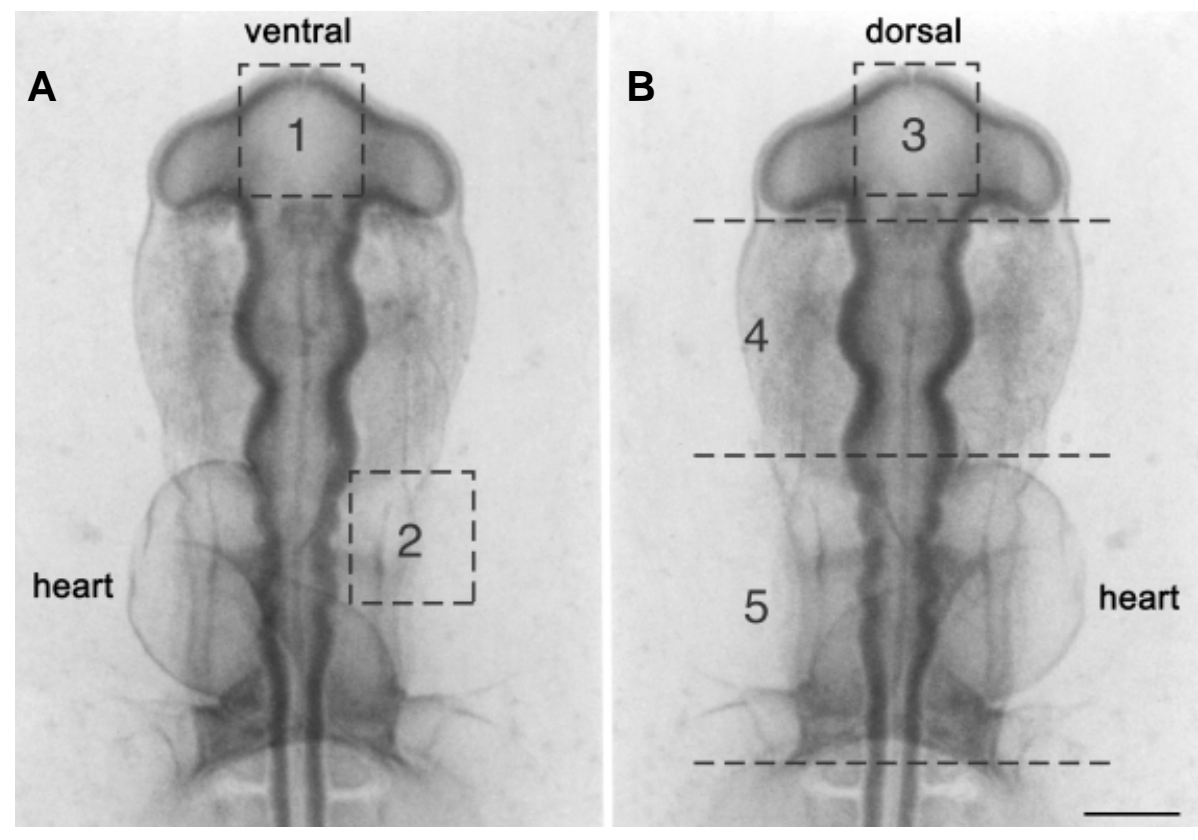

Fig. 5. Spatial pattern of lens specification in stage $\mathbf{1 0}$ embryos. Dotted lines show the boundaries of pieces of ectoderm from the ventral (A) or dorsal (B) regions of head ectoderm that were grown in Medium A for five days as described in the text. Numbers indicate regions of ectoderm cultured and assayed for delta-crystallin expression on immunoblots. See Table I for results. results. 
stained with an antibody to Pax6, the protein co-localized with the mRNA throughout the ectoderm (Fig. 6A). Protein expression was found in nuclei in both the optic vesicles and in a large area of head ectoderm, including the presumptive lens ectoderm (Fig. 6B). We found that regions of head ectoderm that contained some cells expressing Pax6 showed higher levels of specification. In contrast, ectoderm near the heart and over the somites in the trunk region are negative for Pax6 staining and these regions either show very low numbers of positive cultures $(<10 \%)$ or are not specified for lens formation.

\section{Effect of media on lens differentiation}

Because it was possible that either the embryo extract or fetal calf serum in medium A could contain factors that would themselves induce lens differentiation in presumptive lens ectoderm and head ectoderm, we tested these tissues for delta-crystallin synthesis after growth in a variety of less rich media (Fig. 3). For the presumptive lens ectoderm, successful differentiation still occurred in $92 \%$ of the cultures even when embryo extract was omitted from medium B. As the FCS concentration was decreased in media $\mathrm{C}$ and $\mathrm{D}$, there was a slight decline in lens differentiation. Even in the absence of FCS and embryo extract, lens differentiation proceeded in $40 \%$ of the cultures. For ventral head ectoderm, a positive lens response was observed in $84 \%$ of the cultures with the removal of embryo extract from media B. However $100 \%$ of the cultures were positive when FCS was reduced from $15 \%$ to $5 \%$. With further reduction of FCS, the lens response decreased, as it did in presumptive lens ectoderm, yet even in the absence of embryo extract and serum, $50 \%$ of the ventral head ectoderm cultures were positive for delta-crystallin. In all these media, delta-crystallin synthesis by trunk ectoderm cultures was observed only rarely. We conclude that an extensive region of head ectoderm is specified for lens formation at stage 10 , and before, and that specification is not largely dependent on factors in embryo extract or serum. However, we did notice that tissues did not grow as well in the less rich media based on the smaller mass of tissue on a filter at the end of the culture period. In serum-free medium no apparent growth of tissues occurred, and though it might have been preferred to perform further experiments in serum-free medium, the poor growth of tissues precludes more general use of these culture conditions.

\section{Lens induction by the optic vesicle}

The broad area of ectoderm specified for lens formation and the early detection of specification suggested that lens induction begins before optic vesicle formation and occurs in cells that are not in close proximity to it. Yet because of a report in the literature that the optic vesicle is the primary lens inducer (KarkinenJääskeläinen, 1978), we re-examined lens induction by the optic vesicle by following the procedures and culture conditions in this study as closely as possible (Table II). In this series, chick serum was used in the place of FCS because Karkinen-Jääskeläinen noted enhanced lens differentiation in media containing chicken serum. However, we saw no difference in lens differentiation in media containing chicken serum or FCS (data not shown). As a positive control, presumptive lens ectoderm from stage 10 chicken embryos was tested and it differentiated into lenses in close to $100 \%$ of the cultures grown in medium A. As negative controls, both trunk ectoderm from over the somites and optic vesicles were isolated and cultured alone. Neither region was positive for deltacrystallin synthesis on immunoblots (see Fig. 2), nor was lens fiber elongation ever observed under these culture conditions. When stage 10 optic vesicles were combined with stage 10 trunk ectoderm and grown in culture for five days, a positive lens-response was observed only rarely when assayed by polyacrylamide gel electrophoresis and immunoblotting (Table II). When tissues were sectioned and stained with a delta-crystallin antibody, a positive lens response was not observed (data not shown).

Because lens specification has occurred by stage 8 , we tested the nascent optic vesicles from this stage too. In one series of experiments, stage 8 optic vesicles were combined with trunk ectoderm from stage 10 . Only $2 \%$ of the cultures with trunk ectoderm as the responding tissue were positive on immunoblots (Table II).

Earlier, we found that removing embryo extract showed no reduction in successful lens differentiation in culture as long as the FCS concentration was maintained at $15 \%$ (medium B). In another experimental series, we tested the ability of stage 8 optic vesicles

TABLE I

\section{DELTA-CRYSTALLIN SYNTHESIS BY REGIONS OF HEAD ECTODERM GROWN IN MEDIUM A CONTAINING 15\% CHICKEN SERUM AND 20\% EMBRYO EXTRACT}

\begin{tabular}{ccc} 
Region of ectoderm & Lens response lens positive/total & $\%$ positive \\
\hline 1 & $10 / 12$ & 83 \\
2 & $1 / 11$ & 9 \\
3 & $5 / 28$ & 18 \\
4 & $2 / 11$ & 18 \\
5 & $1 / 18$ & 5 \\
presumptive lens ectoderm & $9 / 10$ & 90 \\
\hline
\end{tabular}

See Fig. 5 for the locations of these regions.

TABLE II

DELTA-CRYSTALLIN SYNTHESIS BY CULTURES OF CHICK TISSUES GROWN IN MEDIUM A CONTAINING 15\% CHICKEN SERUM AND 20\% EMBRYO EXTRACT

\begin{tabular}{|c|c|c|c|}
\hline Stage & Tissue(s) cultured & $\begin{array}{c}\text { Lens Response } \\
\text { number positive/total }\end{array}$ & $\%$ positive \\
\hline 10 & presumptive lens ectoderm & $59 / 64$ & 92 \\
\hline 10 & trunk ectoderm & $0 / 22$ & 0 \\
\hline 10 & optic vesicle & $0 / 7$ & 0 \\
\hline 10 & optic vesicle + trunk ectoderm & $0 / 12$ & 0 \\
\hline 8 & optic vesicle + & & \\
\hline 10 & trunk ectoderm & $1 / 52$ & 2 \\
\hline
\end{tabular}

TABLE II

DELTA-CRYSTALLIN SYNTHESIS BY CULTURES OF CHICK TISSUES GROWN IN MEDIUM B CONTAINING 15\% FETAL CALF SERUM AND NO EMBRYO EXTRACT

\begin{tabular}{clcc} 
Stage & Tissue(s) cultured & $\begin{array}{c}\text { Lens Response } \\
\text { number positive/total }\end{array}$ & \% positive \\
\hline 8 & presumptive lens ectoderm & $8 / 9$ & 89 \\
8 & trunk ectoderm & $0 / 14$ & 0 \\
8 & optic vesicle & $0 / 28$ & 0 \\
8 & optic vesicle + trunk ectoderm & $2 / 62$ & 3 \\
8 & optic vesicle + & & \\
10 & trunk ectoderm & $1 / 25$ & 4 \\
\hline
\end{tabular}



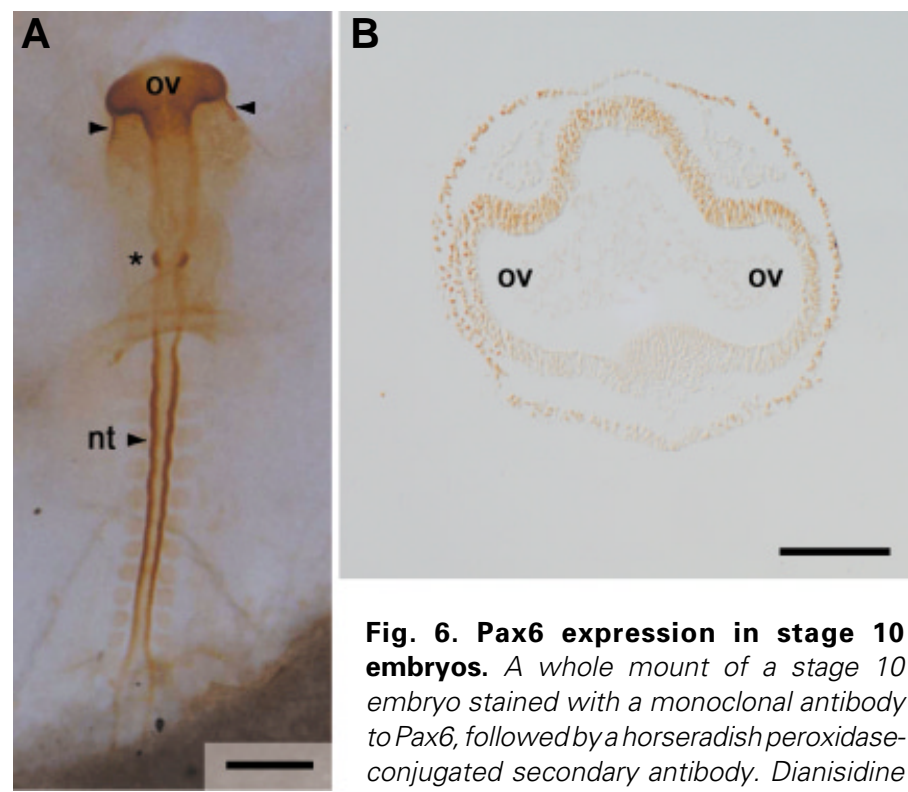

Fig. 6. Pax6 expression in stage 10 embryos. A whole mount of a stage 10 embryo stained with a monoclonal antibody to Pax6, followed by a horseradish peroxidaseconjugated secondary antibody. Dianisidine was added to produce the brown reaction product. At stage 10 (A), staining is visible in the anterior head ectoderm (arrows) and in the optic vesicle (ov) region. Staining is also seen in the third rhombomere (asterisk) and in the neural tube (nt) posterior to the heart. In a transverse section through the optic vesicle, staining is observed in both the neural tissue and in most of the surface ectoderm (B). Scale bar, 0.5 $\mathrm{mm}$ in (A) and 100 $\mu \mathrm{m}$ in (B).

to induce lenses in trunk ectoderm from stage 8 or 10 in medium B. Again a positive response was observed only rarely (Table III).

Even though it has been reported that trunk ectoderm can respond to inductive signals from the optic vesicle, (KarkinenJääskeläinen, 1978), it would be surprising if this ectoderm, because of its late developmental stage, were competent to respond to lens signals, based on previous work with Xenopus (Servetnick and Grainger, 1991). Yet the appropriate test ectoderm is difficult to identify; regions of head ectoderm that are already specified are not good test tissues either because they can differentiate on their own. Therefore, we chose to culture stage 4 epiblast, because this is an early stage when ectoderm is not yet specified (see results above), along with optic vesicles from stage 10. When strips of tissue from each side of the primitive streak were cultured with optic vesicles for five days, all cultures $(n=12)$ were negative by immunoblot analysis. In all of our experiments, the results were far below the $60 \%$ success rate reported previously (KarkinenJääskeläinen, 1978).

\section{Inhibition of lens potential in head ectoderm}

Even though a large region of head ectoderm is specified for lens formation in chick, only a subset of cells express that potential and become a lens in vivo. In the remaining ectodermal cells, the lens potential must be suppressed. We investigated how the position of a lens is finally determined by testing the hypothesis that the underlying head mesenchyme suppresses lens potential in most of the ectoderm. Experiments of two types were conducted. First, a section of tissue, comprised of ectoderm and underlying tissues, was isolated from embryos by making cuts through the midbrain posterior to the optic vesicles and anterior to the heart (Fig. 1A). This region is the source of head ectoderm that is specified for lens formation by stage 10 , as discussed above. These tissue slices with the underlying cells still in contact with head ectoderm, were cultured in Medium A for five days, and then assayed for delta-crystallin. These tissues were never positive (0/ 30). However, when these explants were trypsinized for two minutes (but not dissected) to loosen the association of the ectoderm with the mesenchyme, and then cultured, delta-crystallin was observed in $28 \%$ of the cultures $(n=74)$. As mentioned earlier, when the ventral head ectoderm was trypsinized for two minutes, peeled off of underlying tissue, and cultured alone, delta-crystallin expression was observed in $97 \%$ of the cultures tested (see Fig. 3).

The second type of approach was a recombination experiment. The same piece of midbrain/hindbrain tissue was dissected from stage 10 embryos and placed flat in a culture dish (see Fig. 1C for orientation). Then two cuts in the shape of an $\mathrm{X}$ were made to divide this tissue into four smaller pieces that corresponded to the same regions of ventral-medial and lateral head ectoderm studied earlier by Barabanov and Fedtsova (1982). The upper portion of the $X$ contained the neural tube and was discarded. The lower part of the $X$ (between the lower two white arrowheads in Fig. 1C) was the source of ventral medial ectoderm (adjacent to the gut). The sides of the $X$ (between white arrowheads 1-2 in Fig. 1C) contained the lateral ectoderm. When ectodermal tissues were isolated from underlying mesenchyme with trypsin and grown alone in Medium $A$, both lateral ectoderm and ventral-medial ectoderm showed detectable levels of delta-crystallin expression, in $56 \%$ and $43 \%$ of the cultures, respectively (Fig. 7), though not as high as that observed when entire pieces of ventral head ectoderm were cultured. Yet, when mesenchyme was added back, there was complete inhibition of delta-crystallin synthesis in both regions grown in Medium A, mimicking what occurs in vivo.

The effect of omitting embryo extract on the ability of mesenchyme to suppress lens-forming potential in head ectoderm was tested by

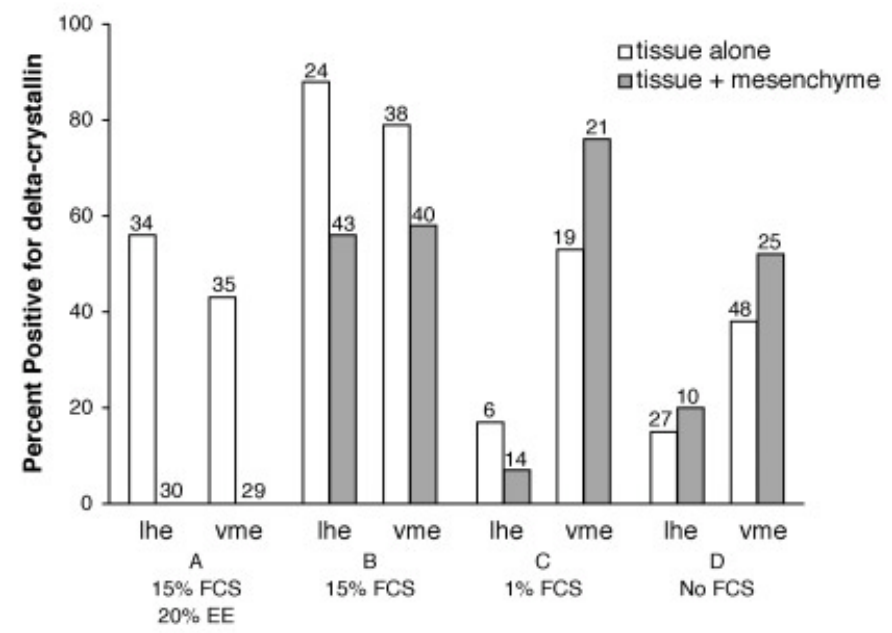

Media for culturing ectodermal tissues

Fig. 7. Effect of head mesenchyme on lens differentiation in two regions of head ectoderm. Ectoderm was removed by trypsinization from ventralmedial ectoderm (vme) or lateral head ectoderm (Ihe) regions of the midbrain and grown in culture alone or combined with adjacent head mesenchyme. Bars show the proportion of cultures that were positive on the basis of deltacrystallin synthesis as assayed on immunoblots. The number above each bar indicates the number of cases scored. 

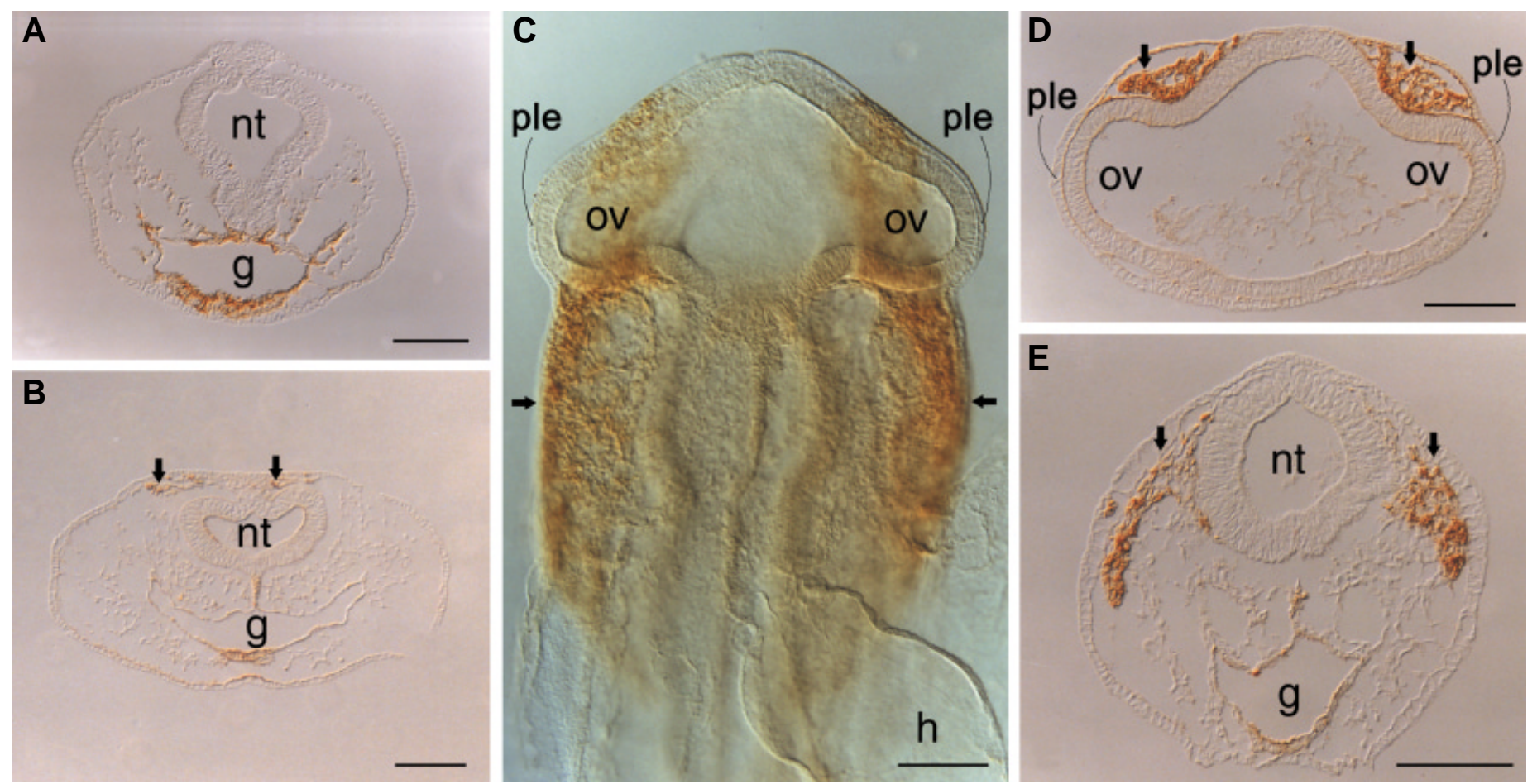

Fig. 8. Migration of neural crest cells in the head mesenchyme of chicken embryos. Whole mounts of embryos were stained the monoclonal antibody HNK-1, followed by a horseradish peroxidase-conjugated secondary antibody. Dianisidine was added to produce the brown reaction product in neural crest cells. Transverse sections of whole mounts of embryos at stage 8 (A) revealed no cephalic neural crest cells on the dorsal side of the neural tube (nt), but staining of other HNK-1 positive cells around the gut (g) is present. By stage 9 (B), migration has begun (arrows). At stage 10, neural crest cells are abundant throughout the head (arrows) as seen on a dorsal view of a whole mount (C). Neural crest cells are absent from the region of contact of the optic vesicle (ov) and presumptive lens ectoderm (ple) on the whole mount and in a transverse section through the optic vesicles (D). Posterior to the optic vesicles (at the level of the arrows in (C), abundant neural crest cells are seen migrating under the lateral head ectoderm on the sides of the embryo and just entering the mesenchyme under the ectoderm on the ventral half (below the level of the neural tube) of the embryo (E). Scale bars, $100 \mu \mathrm{m}$.

growing tissues in Medium B. $88 \%$ of cultures of lateral ectoderm and $79 \%$ of the cultures of ventral-medial ectoderm were positive for synthesis of delta crystallin in the absence of mesenchyme. In contrast to the results with Medium A, mesenchyme did not inhibit the lens response as completely in Medium B. About $60 \%$ of the cultures of lateral and ventral-medial ectoderm were positive when grown in Medium B. However, mesenchyme still suppressed the lens response in these cultures by about $30 \%$ in each case. As the concentration of serum was further reduced, the lens response in the absence of mesenchyme declined, especially in the lateral head ectoderm, and the inhibition by head mesenchyme was lost. In fact, at lower concentrations of serum, mesenchyme appeared to stimulate lens differentiation slightly (Fig. 7). As embryo extract and serum are reduced it would appear that lens differentiation is either no longer responsive to a mesenchymal inhibitor, or that synthesis of the inhibitory factor is reduced.

\section{Location of neural crest cells in chicken embryos}

The lens-forming potential of non-lens head ectoderm is clearly not expressed in vivo. A role for the head mesenchyme in inhibiting this lens potential was demonstrated in our experiments where mesenchyme was recombined with head ectoderm from stage 10 embryos (Fig. 7). It has been shown in transplant experiments that neural crest cells have begun to migrate out from the neural tube and infiltrate head mesenchyme by stage 10 in chicken embryos (Noden, 1975). Therefore, we used the marker HNK-1 to follow neural crest cell migration during the stages we studied earlier. At stage 8, neural crest cells were not detected in the head region (Fig. $8 A$ ). At stage 9, two clusters of cells were observed on either side of the dorsal surface of the neural tube (Fig. 8B). By stage 10, neural crest cells were abundant in the head region (Fig. $8 \mathrm{C}$ ), and were concentrated on the lateral sides of the head right under the head ectoderm (Fig. 8E). Neural crest cells were also present in two clusters along the dorsal surface of each optic vesicle, but were absent from the midline on both the dorsal and ventral surfaces (Fig. 8D). In addition, neural crest cells (and mesenchyme cells in general) were conspicuously absent under the presumptive lens ectoderm (Figs. 8 C,D).

\section{Discussion}

Because the more recent view of lens induction was developed from experiments on a limited number of species of amphibians, it is not known how general the model might be. The purpose of the present study was to test parts of this model in chicken embryos. We present evidence that a large region of head ectoderm is specified for lens formation well before optic vesicle formation and that specification can be detected in a variety of culture media. The optic vesicle was not found to be a potent lens inducer. We show as well that head mesenchyme has a powerful inhibitory effect on the lens potential in lateral and ventral-medial head ectoderm under some culture conditions and that neural crest cells are 
infiltrating the mesenchyme at the time of optic vesicle contact. Taken together, these results are generally consistent with the revised model of lens induction developed for amphibians. However, as discussed below, our data identify some steps in chick lens formation that differ from the amphibian model.

\section{Spatial and temporal specification of lens fate}

We observed that head ectoderm becomes specified for lens formation well before optic vesicle formation and that specification includes ectoderm some distance away from the optic vesicle. The molecular basis for specification is unknown, but our results, coupled with studies on the expression patterns of several genes, are informative. Many genes have been identified that when mutated or knocked out, result in phenotypes affecting eye tissues including the lens (reviewed by Hirsch and Grainger, 2000; Lang, this issue). Some of these genes are expressed very early and are down regulated by the time specification occurs (for example, Sox3 in Xenopus [Zygar et al., 1998]), while others (Sox2 and 3 in chickens [Kamachi et al., 1998] and eyes absentin mice [Xu et al., 1997]) are expressed after optic vesicle contact with the presumptive lens ectoderm. However, the expression pattern of two other genes does occur in tissues at the onset of lens specification. One candidate for a role in specification is the well-studied gene, Pax6, discussed previously. For many regions of the head ectoderm, there is good correspondence between Pax 6 expression and specification. In chick, the larger region of head ectoderm that is specified for lens formation corresponds to a larger domain of Pax6 expression than is seen in Xenopus where the specified area is correspondingly smaller (Zygar etal., 1998). A second gene, Otx2, is up regulated during the bias period in Xenopus (Zygar et al., 1998). In fact, Otx2expression begins just before Pax6expression at the bias stage, and then expression of both genes continues during specification. However, expression of Otx2 and Pax6 are not detectable at the very beginning of bias in Xenopus, so it is likely that other genes are involved in this early event.

\section{Effect of media on lens differentiation}

Because of the wide variety of culture conditions used in past studies, we examined the lens response in different media. The strong lens-forming ability in lens and non-lens head ectoderm was observed in a variety of culture media, ranging from rich media (containing embryo extract and serum), which probably best imitates the environment in the embryo, to media lacking these components. In fact, the lens response in head ectoderm was frequently higher than that observed in the presumptive lens ectoderm. Yet, the response in trunk ectoderm was always very low. Therefore, we believe that we are not observing a widespread activation of lens differentiation because of our culture conditions, but rather we propose that we are seeing a strong tendency for lens formation that is normally repressed in head ectoderm and that is absent in the trunk ectoderm. In addition, lens differentiation does not appear to be dependent on serum and embryo extract components in the culture media used in our experiments even though the richer media do enhance the lens response to some degree.

A somewhat different conclusion was reached in some of the earlier studies of lens formation where the differentiation response of more mature lens tissue was dependent on culture conditions. Several experiments examined the differentiation of fiber cells from lens epithelial cells in vitro. For example, epithelial cells from six day old chicken embyos differentiated into elongated fiber cells (Philpott and Coulombre, 1965) capable of synthesizing crystallins (Piatigorsky et al., 1972) only in the presence of serum, but not in media lacking serum. Yet in our work from younger embryos, the initial response to activate lens differentiation in specified ectoderm does not seem to be greatly dependent on serum conditions.

Several secreted molecules have been shown to have a role in lens induction that could contribute to the enhanced response we see in complex media (see chapters by Lang; Reneker et al., in this issue). For example, two types of bone morphogenetic proteins (BMPs) are critical for lens development. In mice mutant for Bmp4 (Furata and Hogan, 1998) or Bmp7(Wawersik et al., 1999), a lens placode fails to form. The role of BMPs in lens differentiation is unclear. Fibroblast growth factors (FGF) are also synthesized in neural tissue prior to lens formation and Fgf8 can trigger differentiation of lens fiber cells in mouse (Lovicu and Overbeek, 1998) and chicken (Vogel-Hopker et al., 2000) embryos. Finally, retinoic acid is another possible signaling molecule based on the detection of biosynthetic enzymes present during early steps of lens formation in mice (Mic et al., 2000) and developmental effects of retinoid treatments in mouse embryos (Enwright and Grainger, 2000).

\section{Lens induction by the optic vesicle}

Because of the broad and early specification of head ectoderm for lens formation, lens induction must begin before the optic vesicle contacts the presumptive lens ectoderm in chicken embryos. Nevertheless, we attempted to repeat earlier experiments of Karkinen-Jääskeläinen (1978) as closely as possible because they suggest a key role for the optic vesicle. These experiments were designed to test the ability of optic vesicle to induce lenses in trunk ectoderm grown in recombinant cultures. Although a $60 \%$ success rate was reported when both chicken embryos (stages 8 through 11) and mouse embryos (at comparable stages) were examined (Karkinen-Jääskeläinen, 1978), we rarely observed a positive lens response on western blots. Because neither stage 8 nor stage 10 optic vesicles were capable of inducing lens formation in our experiments, it appears that other tissues must be the source of information needed for lens formation. An alternative explanation for our failure to observe lens induction is that the trunk ectoderm at these stages is not competent to respond to a signal from the optic vesicle. Identifying the correct test ectoderm for our experiments is difficult. Large regions of ectoderm that are already specified will proceed on to lens differentiation without further inductive signals. This makes it difficult to evaluate the contribution of the optic vesicle. We have begun to identify the temporal and spatial limits of specification for a lens fate within the head ectoderm with the goal of finding a region of ectoderm to test the contribution of the optic vesicle in lens induction. What we have shown in the present study is that the optic vesicle cannot induce a lens in trunk ectoderm as others have claimed, nor does unspecified epiblast tissue respond. Yet the epiblast along the primitive streak may not be a satisfactory test tissue either and anterior epiblast may be preferred. However, the boundaries between neural tissue, presumptive lens tissue, and other head ectoderm are hard to distinguish in chicken embryos. This difficulty highlights the advantages of studying early aspects of lens induction in Xenopus, where the embryos are larger and the landmarks between neural and non-neural ectoderm are clearer at early developmental stages. 
While it is possible that differences in the source of serum and embryo extract could account for our failure to replicate KarkinenJääskeläinen's results, there are other differences that might be important as well. Two major differences between our assay and the one used by Karkinen-Jääskeläinen were the antibodies used and her reliance solely on immunofluorescence. The antibodies used were prepared against total lens protein, whereas our antibody was prepared against purified delta-crystallin (D. Beebe, personal communication). It is possible that the antibody used by KarkinenJääskeläinen recognized an antigen that is not specific to the lens. Finally, if some ectoderm remained attached to the optic vesicle after enzyme treatment, then this ectoderm could be the source of the positive results observed previously (Karkinen-Jääskeläinen, 1978). While a host and donor marking scheme was used in some of the experiments by Karkinen-Jääskeläinen, the method was not used in all cases and appears not to have been used simultaneously with a marker for lens differentiation (i.e. the lens antibody).

\section{Early lens-inducing tissues}

If the optic vesicle is not the source of the primary lens-inducing signal in the chick, what tissues are responsible? Whether there are earlier signals emanating from the neural plate, or possibly from underlying tissues (as suggested from amphibian work; Grainger, 1996) is not clear from our studies. Experiments by Jorquera et al. (1989) suggested that early presumptive cardiac mesenchyme may be a lens inducer. When stage 4 epiblast was cultured alone, no lens response was observed (similar to our specification experiments reported above). However, when stage 4 epiblast was cultured with cardiac mesenchyme from the same stage, lenses were observed in about half of their cultures. However, in most of these positive cultures, neural tissue was observed too (Jorquera et al., 1989). This latter observation raises the possibility that neural tissue, perhaps presumptive eye tissue, is actually the source of the lens-inducing signal seen in these experiments.

\section{Inhibitory tissue interactions in lens formation}

Our specification experiments showed that an extensive region of head ectoderm is capable of lens differentiation when separated from underlying mesenchyme and grown in vitro. However, this potential is suppressed in most of the head ectoderm in vivo; only the presumptive lens ectoderm differentiates into a lens. Inhibition of the lens potential was also observed in vitro when underlying mesenchyme was left attached to the ectoderm or recombined with lateral or ventral-medial ectoderm. We found that the HNK-1 positive cells have begun to migrate under lateral head ectoderm by stage 10, agreeing well with Noden's work neural crest cell migration (1975). He used transplants of ${ }^{3} \mathrm{H}$-thymidine labeled tissue and observed that neural crest cells had migrated under head ectoderm by stage 12-13. Therefore, it seems likely that neural crest cells are in position to be the source of the inhibitory signal that suppresses the lens potential in both the lateral and ventral-medial regions of the head ectoderm.

We also observed that embryo extract was important for preserving the inhibitory effect of mesenchyme in culture. This result suggests that the embryo extract might contain a factor that contributes to the inhibition of lens differentiation when mesenchyme is present. In their studies of lens cell proliferation, Hyatt and Beebe (1993) identify a factor that might be responsible for this effect. One well-known characteristic of lens cells is their withdrawal from the cell cycle as part of differentiation (Modak et al., 1968). Hyatt and Beebe found a factor in chick serum that promotes cell division and blocks cell elongation characteristic of lens differentiation. This factor had its highest activity in serum from 10-day embryos, the same age we used to prepare embryo extract. It is possible that this factor is contributing to the inhibition of delta-crystallin synthesis in cultures grown in medium A containing embryo extract. Potts et al. (1998) have shown that proliferation of embryonic lens epithelial cells can be maintained in vitro by an unidentified factor found in serum that stimulated STAT phosphorylation, suggesting the involvement of the Jak-STAT pathway in regulating this balance between proliferation and differentiation. A growing list of growth factors, growth factor receptors and components of other signal transduction pathways appear to be part of controlling this balance as well (see chapters by Beebe et al., Chu et al. and Reneker et al. in this issue.)

While the observation that the other media do not lead to full inhibition by mesenchyme might imply that this inhibition is not a primary role for these cells, the fact that media containing embryo extract shows a strong inhibitory effect may imply that this is simply the medium that best recapitulates in vivoconditions. Further study will be required to establish in a definitive way which of these interpretations is correct.

\section{What is the role of the optic vesicle?}

Our results do not support a model in which the optic vesicle is the primary inducer of the lens. However, it is possible that the optic vesicle contributes to the final phases of lens induction. Indeed there is evidence from amphibians that transplantation of the optic vesicle to regions of non-lens head ectoderm can induce lenses in already biased, but not specified, ectoderm (Grainger et al., 1997). In the chick, an anterior-posterior gradient in the lens response of the ectoderm was observed by Alexander (1937), in his transplant experiments on stage 8 and 9 hosts that may reflect similar properties in the chick. A higher percentage of positive lens responses was seen when optic vesicles were transplanted under ectoderm surrounding the eye, than when transplanted under head and neck ectoderm. Transplants under trunk ectoderm were negative. Recent results in Xenopus indicate that during normal development the optic vesicle may play a key role in converting specified ectoderm to determined ectoderm, that is, establishing the final, irreversible commitment of lens ectoderm to a lens fate (Jin and Grainger, unpublished).

The tight association of the presumptive lens ectoderm and optic vesicle suggests that an additional important role for the optic vesicle may be to block neural crest cells and other mesenchyme from entering this region thereby suppressing the inhibitory effects of mesenchymal cells. Whether the optic vesicle provides a positive signal in addition to preventing the inhibition from adjacent mesenchyme is as yet unclear.

The final role for the optic vesicle comes from evidence that the neural retina (derived from the optic vesicle) is needed for differentiation of lens fiber cells (Coulombre and Coulombre, 1963). They showed that if the position of a lens from a five-day chicken embryo is reversed in the eye so that the anterior epithelial cells face the vitreous humor and neural retina, epithelial cells will convert to fiber cells. While a number of growth factors appear to play a role in lens differentiation, members of the fibroblast growth factor family produced by the neural retina, are particularly important 
based on studies in both mouse (Robinson et al., 1998; Stolen and Griep, 2000) and chicken (McAvoy and Chamberlain, 1989; Le and Musil, 2001) embryos.

\section{Does the revised model of lens induction apply to chicken embryos?}

Based on what information we have on lens induction from various organisms, as well as the conservation of eye regulatory genes in a large number of species (reviewed by Hirsch and Grainger, 2000), it seems plausible that features of eye formation, including lens induction, might be fundamentally similar in animals. The results presented in this paper suggest that in chicken embryos, the optic vesicle is not the primary inducer, but that earlier tissue interactions are necessary for lens formation, as in amphibians, though the source of the early signal is as yet uncertain in the chick.

While a lens-forming bias is established in large regions of head ectoderm in amphibians (Grainger et al., 1997), the region of lens specification is restricted to the presumptive lens area. The situation is somewhat different in the chick in that specification occurs much earlier than in Xenopus, where this event is coincident with contact from the optic vesicle (Henry and Grainger, 1990). We also have evidence that non-lens head ectoderm between the optic vesicles and the heart remains specified for about 12 hours after optic vesicle contact, though stage 13 (G. Syed and C. Sullivan, unpublished). Therefore, the inhibition by the mesenchyme that we observe is an event that must still be occurring in vivo well after specification is first detected. Whether the broader region of specified ectoderm in the chick results from a stronger early inductive signal, or a weaker inhibitory signal, relative to amphibians, remains to be determined.

Our results are consistent with a role for head mesoderm in suppressing lens specification in head ectoderm. As noted earlier, data from amphibians has suggested a role for neural crest cells in inhibiting the lens potential in head ectoderm (von Woellwarth, 1961). Involvement of the neural crest is also suggested from studies of embryos with mutations in the Pax6 gene in rats (Matsuo et al., 1993). Lenses do not form in these mutant animals in which neural crest migration is disturbed. Interestingly, neural crest cells that are normally destined for the olfactory area move into the eye region. If the neural crest cells are the source of the inhibitory signal, they may contribute to the absence of lenses in cases where neural crest cells accumulate in the eye region of mutant rats. Recently, Enwright and Grainger (unpublished) have also shown that head mesoderm in mouse can inhibit the lens-forming ability of the presumptive lens ectoderm in this species. Our studies on the chick suggest that neural crest cells may contribute to the inhibitory effect that we see, but further work is needed to clarify their role.

\section{Materials and Methods}

\section{Embryos}

Fertile White Leghorn eggs (CBT Farms, Chestertown, MD) were incubated at $38^{\circ} \mathrm{C}$ in a humidified incubator for the appropriate time to reach desired developmental stages (Hamburger and Hamilton, 1951). Each egg was rinsed in $70 \%$ ethanol, dried, then opened into sterile phosphatebuffered saline (PBS). Embryos were collected as described previously (Sullivan et al., 1991a). Briefly, embryos were removed from the surface of the yolk, then excess tissue was trimmed using 27 gauge needles attached to $1 \mathrm{ml}$ syringes. Most of our experiments were conducted with stage 10 (10-
12 pairs of somites) embryos that were cut at four locations (see dotted lines in Fig. $1 \mathrm{~A}$ ) to isolate the forebrain containing the two optic vesicles, the remainder of the head (midbrain and hindbrain), the heart region, and the trunk. Then surface ectoderm was separated from underlying tissues following a brief incubation in 3\% trypsin-pancreatin in PBS on ice (KarkinenJääskeläinen, 1978). Activity of the trypsin was stopped by transferring the tissues to $20 \%$ chicken serum in PBS. Tissues remained in the serum solution for 10-15 minutes while being dissected. The lens-forming potential of different regions of ectoderm was studied as described in the next section. Presumptive lens was isolated from over the optic vesicles (Fig. 1B) and trunk ectoderm was collected from over the somites (Fig. 1D). In our previous experiments (Sullivan et al., 1998), an entire strip of head ectoderm was dissected from the surface of the midbrain. In this study, most of our experiments used a smaller piece of head ectoderm from the ventral half of the midbrain (Fig. 1C).

\section{Tissue culture}

Pieces of tissue were cultured on small squares of Millipore or Nuclepore filters supported on a wire mesh screen and placed in a $35 \mathrm{~mm}$ culture dish as described previously (Sullivan et al., 1998). The typical culture medium (medium A) was Basal Medium Eagle (BME) supplemented with essential and non-essential amino acids (GibcoBRL), 20\% embryo extract (EE) prepared according to Peterson and Grainger (1985), and 15\% heatinactivated chicken serum (CS) or fetal calf serum (FCS) after KarkinenJääskeläinen (1978). In other media the embryo extract was omitted. Medium B contained 15\% FCS, medium C contained 5\% FCS, medium D contained $1 \% \mathrm{FCS}$, and medium E contained $0 \% \mathrm{FCS}$. Culture dishes were set up and the media pre-equilibrated in a $37^{\circ} \mathrm{C}$ incubator with $5 \% \mathrm{CO}_{2}$. The medium was changed on the second or third day and tissues were typically cultured for five days.

\section{Histology and immunofluorescence}

Cultures were harvested and placed directly in cold Bouin's fixative overnight. Tissues were dehydrated through alcohol, cleared in xylene and embedded in paraplast. $7 \mu \mathrm{m}$ sections were cut on a microtome and were processed by routine methods (Humason, 1979). To localize sites of deltacrystallin accumulation, sections were incubated for an hour with a 1:2000 dilution of a polyclonal antibody to purified delta-crystallin prepared in rabbits as described previously (Sullivan et al., 1998). Slides were rinsed in 1XPBS then stained with a 1:250 dilution of fluorescein-conjugated goatanti-rabbit (American Qualex) secondary antibody, also for an hour at $37^{\circ} \mathrm{C}$. Sections were viewed with an Olympus BX-60 photomicroscope equipped for epifluorescence.

\section{Polyacrylamide gel electrophoresis and immunoblotting}

Most cultures were analyzed for delta-crystallin synthesis by polyacrylamide gel electrophoresis and immunoblotting. An entire culture was placed in 1X Laemmli (1970) sample buffer (8\% sucrose; $5 \% \beta$ mercaptoethanol; $2 \%$ sodium dodecyl sulfate; $62.5 \mathrm{mM}$ Tris $\mathrm{HCl}, \mathrm{pH} 6.8$; $0.2 \mathrm{mg}$ bromophenol blue). Samples were boiled for 5 minutes and stored at $-20^{\circ} \mathrm{C}$ until analyzed by polyacrylamide gel electrophoresis as described below.

Proteins were separated by polyacrylamide gel electrophoresis in the presence of sodium dodecyl sulfate (Laemmli, 1970) on 10\% gels. Gels were then prepared for electroblotting to nitrocellulose (Towbin et al., 1979). After transfer, nitrocellulose was processed as described previously (Sullivan et al., 1998). The primary antibody to delta-crystallin was used at a 1:1000 dilution and was applied for 2 hours at $37^{\circ} \mathrm{C}$. The secondary antibody was a goat anti-rabbit IgG conjugated to horseradish peroxidase (American Qualex) with dianisidine as the substrate.

\section{Whole mount immunocytochemistry}

Chicken embryos were processed for whole mount staining using a modification (Lefcort, personal communication) of the procedure of Dent $e t$ al., (1989). Embryos were fixed in methanol:dimethylsulfoxide (4:1) and 
bleached as described. Specimens were rehydrated through methanol to PBS, then incubated in two changes of PBS containing $10 \%$ goat serum and $0.1 \%$ Triton X-100 (PBSGST) at room temperature for an hour each. Specimens were incubated overnight at $4^{\circ} \mathrm{C}$ in a $1: 10$ dilution of mouse hybridoma supernatant (American Type Tissue Collection) from the neural crest marker HNK-1 (Vincent et al., 1983) in PBSGST or in a 1:5 dilution of mouse hybridoma supernatant (Developmental Studies Hybridoma Bank) to Pax6. The next day, specimens were washed five times in PBSGST; the first two washes were at $4^{\circ} \mathrm{C}$ and the others at room temperature. Embryos were incubated overnight at $4^{\circ} \mathrm{C}$ in a horseradish peroxidase-conjugated goat anti-mouse secondary antibody (American Qualex) diluted 1:250 in PBSGST. Specimens were washed five times as above in PBSGST and were washed a final time in PBS containing $0.2 \%$ bovine serum albumin and $0.1 \%$ Triton $X-100$ (PBT). Color was developed using $0.03 \%$ diaminobenzidine in PBT for 20 minutes, then adding hydrogen peroxide to a final concentration of $0.03 \%$. The reaction was stopped by replacing the substrate with $50 \%$ ethanol. Embryos were dehydrated through a methanol series and cleared in benzyl alcohol:benzyl benzoate (1:2). Stained whole mount specimens were embedded in Paraplast and sectioned by routine procedures.

\section{Acknowledgments}

The authors thank Dr. David Beebe, Washington University, for supplying the delta-crystallin antibody used in these studies and to Dr. Frances Lefcort of Montana State University for supplying the anti HNK-1 antibody. We also thank Stephanie Peterson of Grinnell College for preparing the figures. This research was supported by NIH grants EY06675 and EY10283 to RMG. CHS was supported by a Faculty Study Leave and research awards from Grinnell College. This article is contribution No. 3 from the Barr Laboratory of DevelopmentalBiology and CellularNeuroscience at Grinnell College.

\section{References}

AGATA, K., YASUDA, K. and OKADA, T.S. (1983). Gene coding for a lens-specific protein, delta-crystallin, is transcribed in non-lens tissues of chicken embryos. Dev. Biol. 100: 222-226.

ALEXANDER, L.E. (1937). An experimental study of the role of optic cup and overlying ectoderm in lens formation in the chick embryo. J. Exp. Zool. 75: 41-73.

ASHERY-PADAN, R. and GRUSS, P. (2001). Pax6 lights up the way for eye development. Curr. Opinion in Cell Biology 13: 706-714.

BARABANOV, V.M. and FEDTSOVA, N.G. (1982). The distribution of lens differentiation capacity in the head ectoderm of chick embryos. Differentiation 21: 183190.

BRONNER-FRASER, M. (1996). Methods in Avian Embryology. Academic Press, San Diego.

COULOMBRE, J.L. and COULOMBRE, A.J. (1963). Lens development: fiber elongation and lens orientation. Science 142: 1489-1490.

DENT, J.A., POLSON, A. G. and KLYMKOWSKY, M. W. (1989). A whole-mount immunocytochemical analysis of the expression of the intermediate filament protein vimentin in Xenopus. Development 105: 61-74.

ENWRIGHT, J.F. and GRAINGER, R.M. (2000). Altered retinoid signaling in the heads of Small eye mouse embryos. Dev. Biol. 221: 10-22.

FUHRMANN, S., LEVINE, E.M. and REH, T.A. (2000). Extraocular mesenchyme patterns the optic vesicle during early eye development in the embryonic chick. Development 127: 4599-4609.

FUJIWARA, M. UCHIDA, T. OSUMI-YAMASHITA, N. and ETO, K. (1994). Uchida rat (rSey): A new mutant rat with craniofacial abnormalities resembling those of the mouse Seymutant. Differentiation 57: 31-38.

FURUTA, Y. and HOGAN, B.L.M. (1998). BMP4 is essential for lens induction in the mouse embryo. Genes Dev. 12: 3764-3775.

GEHRING, W.J. and IKEO, K. (1999). Pax 6. Mastering eye morphogenesis and eye evolution. Trends in Genetics 15: 371-377.
GRAINGER, R.M. (1992). Embryonic lens induction: shedding light on vertebrate tissue determination. Trends in Genetics 8: 349-355.

GRAINGER, R.M. (1996). New perspectives on embryonic lens induction. Semin. Cell Dev. Biol:: 149-155.

GRAINGER, R.M., HENRY, J. J. and HENDERSON, R.A. (1988). Reinvestigation of the role of the optic vesicle in embryonic lens induction. Development102:517-526.

GRAINGER, R.M., MANNION, J.E., COOK, T.L., JR. and ZYGAR, C.A. (1997). Defining intermediate stages in cell determination: acquisition of a lens-forming bias in head ectoderm during lens determination. Dev. Genetics 20: 246-257.

HALDER, G., CALLAERTS, P. and GEHRING, W.J. (1995). Induction of ectopic eyes by targeted expression of the eyelessgene in Drosophila. Science267: 1788-1792.

HAMBURGER, V. and HAMILTON, H.L. (1951). A series of normal stages in the development of the chick embryo. J. Morph. 88: 49-92.

HENRY, J.J. and GRAINGER, R.M. (1987). Inductive interactons in the spatial and temporal restriction of lens-forming potential in embryonic ectoderm of Xenopus laevis. Dev. Biol. 124: 200-214.

HENRY, J.J. and GRAINGER, R.M. (1990). Early tissue interactions leading to embryonic lens formation in Xenopus laevis. Dev. Biol. 141: 149-163.

HIRSCH, N. and GRAINGER, R.M. (2000). Induction of the lens. In Vertebrate Eye Development(E. Fini, Ed.), pp. 51-68. Springer-Verlag, Berlin/Heidelburg.

HUMASON, G.L. (1979). "Animal Tissue Techniques" Freeman, San Francisco.

HYATT, G.A. and BEEBE, D. C. (1993). Regulation of lens cell growth and polarity by an embryo-specific growth factor and by inhibitors of lens cell proliferation and differentiation. Development 117: 701-709.

JACOBSON, A. (1966). Inductive processes in embryonic development. Science152 25-52.

JEANNY, J-C., BOWER, D.J., ERRINGTON, L.H., MORRIS, S. and CLAYTON, R.M. (1985). Cellular heterogeneity in the expression of the delta-crystallin gene in nonlens tissues. Dev. Biol. 112: 94-99.

JORQUERA, B., GOICOECHEA, O. and MOLINARI, E. (1989). Diferenciacion de cristalinos en explantes de embrion de pollo en ausencia de hipoblasto y vesicula optica. Anat. Histol. Embryol. 18: 32-44.

KAMACHI, Y., UCHIKAYA, M., COLLIGNON, J., LOVELL-BADGE, R. and KONDOH, H. (1998). Involvement of Sox 1, 2 and 3 in the early and subsequent molecular events of lens induction. Development 125: 2521-2532.

KARKINEN-JÄÄSKELÄINEN, M. (1978). Permissive and directive interactions in lens induction. J. Embryol. Exp. Morph. 44: 167-179.

LAEMMLI, U.K. (1970). Cleavage of structural proteins during assembly of the head of bacteriophage T4. Nature 227: 680-685.

LE, A.-C.N. and MUSIL, L.S. (2001). FGF signaling in chick lens development. Dev. Biol. 233: 394-411.

LI, H.-S., YANG, J.-M., JACOBSON, R.D., PASKO, D. and SUNDIN, O. (1994). Pax6 is first expressed in a region of ectoderm anterior to the early neural plate: implications for stepwise determination of the lens. Dev. Biol. 162: 181-194.

LI, H.-S., TIERNEY, C., WEN, L, WU, J.Y. and RAO, Y. (1997). A single morphogenetic field gives rise to two retina primordial under the influence of the prechordal plate. Development 124: 603-615.

LOVICU, F.J. and OVERBEEK, P.A. (1998). Overlapping effects of different members of the FGF family on lens fiber differentiation in transgenic mice. Development 125 : 3365-3377.

MCAVOY, J.W. and CHAMBERLAIN, C.G. (1989). Fibroblast growth factor (FGF) induces different responses in lens epithelial cells depending on its concentration. Development 107: 221-228.

MATSUO, T., OSUMI-YAMASHITA, N., NOJI, S., OHUCHI, H., KOYAMA, E., MYOKAI, F., MATSUO, N., TANIGUCHI, S., DOI, H., ISEKI, S., NINOMIYA, Y., FUJIWARA, M., WATANABE, T. and ETO, K. (1993). A mutation in the Pax-6 gene in rat small eye is associated with impaired migration of midbrain crest cells. Nat. Genet. 3: 299-304.

MCKEEHAN, M.S. (1951). Cytological aspects of embryonic lens induction in the chick. J. Exp. Zool. 117: 31-64.

MIC, F.A., MOLOTKOV, A., FAN, X., CUENCA, A.E. and DUESTER, G. (2000). RALDH3, a retinaldehyde dehydrogenase that generates retinoic acid, is expressed in the ventral retina, otic vesicle and olfactory pit during mouse development. Mech. Dev. 97: 227-230. 
MODAK, S.P., MORRIS, G. and YAMADA, T. (1968). DNA synthesis and mitotic activity during early development of chick lens. Dev. Biol. 17: 544-561.

NODEN, D.M. (1975). An analysis of the migratory behavior of avian cephalic neural crest cells. Dev. Biol. 42: 106-130.

PETERSON, C.A. and GRAINGER, R.M. (1985). Differentiation of embryonic chick feather-forming and scale-forming tissues in transfilter cultures. Dev. Biol. 111: 825.

PHILPOTT, G.W. and COULOMBRE, A.J. (1965). Lens development II. The differentiation of embryonic chick lens epithelial cells in vitroand in vivo. Exp. CellRes. 38: 635-644.

PIATIGORSKY, J., WEBSTER, H. and CRAIG, S.P. (1972). Protein synthesis and ultrastructure during the formation of embryonic chick lens fibers in vitro and in vivo. Dev. Biol. 27: 176-189.

POTTS, J.D., KORNACKER, S. and BEEBE, D.C. (1998). Activation of the Jak-STATsignalling pathway in embryonic lens cells. Dev. Biol. 204: 277-292.

ROBINSON, M.L., OHTAKA-MARUYAMA, C., CHAN, C.-C., JAMIESON, S., DICKSON, C., OVERVEEK, P.A. and CHEPELINSKY, A.B. (1998). Disregulation of ocular morphogenesis by lens-specific expression of FGF-3/Int-2 in transgenic mice. Dev. Biol. 198: 13-31.

SAHA, M.S., SPAAN, C.L. and GRAINGER, R.M. (1989). Embryonic lens induction: more than meets the optic vesicle. Cell Diff. and Dev. 28: 153-172.

SERVETNICK, M. and GRAINGER, R.M. (1991). Changes in neural and lens competence in Xenopus ectoderm: evidence for an autonomous developmental timer. Development 112: 177-188.

SPEMANN, H. (1938). Embryonic Development and Induction. Yale University Press, New Haven.

STOLEN, C.M. and GRIEP, A.E. (2000). Disruption of lens fiber cell differentiation and survival at multiple stages by region-specific expression of truncated FGF receptors. Dev. Biol. 217: 205-220.
SULLIVAN, C.H., HART, J.P. and KRAMER, J. (1991a). The pattern of protein and glycoprotein synthesis in presumptive lens and non-lens ectoderm of the chicken embryo. Roux's Arch. Dev. Biol. 200: 38-44.

SULLIVAN C.H., O'Farrell, S. and GRAINGER, R.M. (1991b). Delta-crystallin gene expression and patterns of hypomethylation demonstrate two levels of regulation for the delta-crystallin genes in embryonic chick tissues. Dev. Biol. 145: 4050.

SULLIVAN, C.H., MARKER, P.C., THORN, J.M. and BROWN, J.D. (1998). Reliability of delta-crystallin as a marker for studies of chick lens induction. Differentiation 64: 1-9.

TOWBIN, H. STAEHELIN, T. and GORDON, J. (1979). Electrophoretic transfer of proteins from polyacrylamide gels to nitrocellulose sheets: procedure and some applications. Proc. Natl. Acad. Sci. USA 76: 4350-4354.

VINCENT, M., DUBAND, J-L. and THIERY, J.P. (1983). A cell surface determinant expressed early on migrating avian neural crest cells. Dev. Brain Res. 9: 235238.

VOGEL-HÖPKER, A., MOMOSE, T., ROHRER, H., YASUDA, K., ISHIHARA, L. and RAPAPORT, D.H. (2000). Multiple functions of fibroblast growth factor-8 (FGF-8) in chick eye development. Mech. Dev. 94: 25-36.

VON WOELLWARTH, C. (1961). Die rolle des neuralleistenmaterials und der temperatur bei der determination der augenlinse. Embryologia 6: 219-242.

WAWERSIK, S., PURCELL, P., RAUCHMAN, M., DUDLEY, A.T., ROBERTSON, E.J. and MAAS, R. (1999). BMP7 acts in murine lens placode development. Dev. Biol. 207: 176-188.

XU, P.-X., WOO, I., HER, H., BEIER, D.R. and MAAS, R.L. (1997). Mouse Eya homologues of the Drosophila eyes absentgene require Pax6for expression in lens and nasal placodes. Development 124: 219-231.

ZYGAR, C.A., COOK, JR T. L. and R.M. GRAINGER. (1998). Gene activation during early stages of lens induction in Xenopus. Development 125: 3509-3519. 